\title{
MAXIMAL ALGEBRAS OF CONTINUOUS FUNCTIONS
}

\section{HENRY HELSON AND FRANK QUIGLEY}

We are interested in the following general problem: given a compact Hausdorff space $S$, and the algebra $C(S)$ of all complex-valued continuous functions on $S$, to describe the subalgebras of $C(S)$ closed in the topology of uniform convergence. In several recent papers John Wermer has considered some difficult special cases. The theorems presented here were suggested by certain of Wermer's results.

Let $S$ be the unit circle. Wermer has found a family of subalgebras of $C(S)$ which are maximal among all closed subalgebras of $C(S)$ $[1 ; 2 ; 3]$. These algebras can roughly be described in the following way: let a small copy of the circle be smoothly inscribed on a closed Riemann surface; consider the functions analytic on the part of the surface outside the circle and continuous on the circle as well. Functions of this type clearly form a closed subalgebra of $C(S)$, which turns out to be maximal.

This note is a beginning in the opposite direction. Given a compact space $S$ and a maximal closed subalgebra $\mathfrak{A}$ of $C(S)$, we can prove that $\mathfrak{A}$ shares a number of properties of algebras whose elements are analytic functions. Since we require no other hypotheses on $S$, this analysis can be carried only to a certain point, and complete information for any particular space $S$ will depend on subtler notions than we use.

We assume, then, that $C(S)=\mathbb{E}$ is the algebra of continuous functions on the compact Hausdorff space $S$, and that $\mathfrak{A}$ is a subalgebra of $\mathfrak{E}$, closed in the uniform topology and contained in no other proper closed subalgebra of $\mathfrak{E}$. If it happens that $\mathfrak{A}$ is an ideal in $\mathfrak{E}$, it is clearly a maximal ideal and consists of all the continuous functions vanishing at a fixed point of $S$. (Conversely every maximal ideal is a maximal closed subalgebra.) We have in fact

Theorem 1. Either $\mathfrak{A}$ is a maximal ideal in $\mathfrak{S}$, or $\mathfrak{A}$ contains the scalars.

If $\mathfrak{A}$ does not contain the scalars, form a larger subalgebra $\mathfrak{A}^{\prime}$ whose elements are all the functions $\phi+\lambda(\phi \in \mathfrak{A}, \lambda$ complex). Since $\mathfrak{A}$ is maximal, $\mathfrak{A}^{\prime}$ must be dense in $\mathfrak{E}$. Evidently $\mathfrak{A} \cdot \mathfrak{A}^{\prime} \subset \mathfrak{A},{ }^{1}$ and since $\mathfrak{A}$ is closed, $\mathfrak{A} \cdot \mathfrak{S} \subset \mathfrak{A}$. That is, $\mathfrak{A}$ is a proper closed ideal in $\mathfrak{E}$ and so is a maximal ideal, as we had to show.

Received by the editors February 28, 1956.

1 The set of products of elements of $\mathfrak{A}$ by elements of $\mathfrak{I}^{\prime}$ is denoted by $\mathfrak{A} \cdot \mathfrak{A}$. 
The maximal ideals $\mathfrak{A}$ are not of interest from our point of view, and by excluding them we may assume from this point on that $\mathfrak{A}$ contains scalars.

THEOREM 2. Either $\mathfrak{A}$ separates points on $S$, or $\mathfrak{A}$ consists exactly of all functions $f$ in $\sqrt{ }$ such that $f(p)=f(q)$ for a certain pair of distinct points $p$ and $q$.

This follows directly from the fact that $\mathfrak{A}$ is maximal. We now assume further that $\mathfrak{A}$ separates points on $S$.

The next theorem will reduce the study of the remaining maximal algebras to those without divisors of zero.

THEOREM 3. There is a nonempty closed subset $S_{0}$ of $S$ such that $\mathfrak{A}$ contains the set $\mathfrak{A}_{0}$ of all functions in $\mathfrak{C}$ vanishing on $S_{0}, \mathfrak{A} / \mathfrak{H}_{0}$ is a maximal closed subalgebra of $\mathfrak{E} / \mathfrak{A}_{0}$, and $\mathfrak{A} / \mathfrak{A}_{0}$ has no divisors of zero.

From the proof it will be clear that there is a unique set $S_{0}$ with the properties asserted; $\mathfrak{A}$ has divisors of zero if and only if $S_{0}$ is not $S$.

Lemma. If $\mathfrak{A}$ has divisors of zero, then $\mathfrak{A}$ contains a proper closed ideal of $\mathfrak{S}$. In fact, if $\phi, \psi \in \mathfrak{A}$ and $\phi \cdot \psi=0$, then either $\phi \cdot \mathfrak{C} \subset \mathfrak{A}$ or $\psi \cdot(\mathfrak{S} \subset \mathfrak{A}$.

Suppose indeed that $f \in \mathbb{C}$ but $\phi \cdot f \notin \mathcal{A}$ (so that we do not have $\phi \cdot(\mathfrak{C} \mathfrak{A})$. Because $\mathfrak{A}$ is maximal in $\mathfrak{S}$, the functions of the form

$$
P=\alpha_{0}+\alpha_{1}(\phi f)+\alpha_{2}(\phi f)^{2}+\cdots+\alpha_{n}(\phi f)^{n} \quad\left(\alpha_{j} \in \mathfrak{A}\right)
$$

form a dense subset of $\mathcal{E}$. Since $\phi \cdot \psi=0$, we have $\psi \cdot P=\psi \cdot \alpha_{0} \in \mathfrak{A}$ for each function $P$. Since $\mathfrak{A}$ is closed, it follows that $\psi \cdot \mathfrak{C} \subset \mathfrak{A}$, as we had to prove.

Proceeding to the proof of the theorem, we denote by $\mathfrak{A}_{0}$ the set of all $\phi$ in $\mathfrak{A}$ such that $\phi \cdot \mathfrak{C} \subset \mathfrak{A}$. If $\mathfrak{A}$ has divisors of zero, $\mathfrak{A}_{0}$ contains other functions than zero by the lemma. Clearly $\mathfrak{A}_{0}$ is a closed ideal of $\mathfrak{C}$, and so consists of all the functions in $\mathbb{S}$ vanishing on a certain nonempty subset $S_{0}$ of $S$. If there were a closed algebra properly between $\mathfrak{A} / \mathfrak{A}_{0}$ and $\mathfrak{S} / \mathfrak{A}_{0}$, we could construct an algebra properly between $\mathfrak{A}$ and $\mathfrak{S}$, which is impossible.

Finally we have to show that $\mathfrak{A} / \mathfrak{A}_{0}$ has no divisors of zero. Each element of $\mathfrak{E} / \mathfrak{A}_{0}$ can be identified with a function on $S_{0}$, so that $\mathfrak{C} / \mathfrak{A}_{0}$ is isomorphic with $C\left(S_{0}\right)$. Under this correspondence $\mathfrak{A} / \mathfrak{A}_{0}$ is represented as a subalgebra of $C\left(S_{0}\right)$ which has been asserted to be maximal, and which obviously contains constants and separates points on $S_{0}$. Applying the lemma once more, we may finish the proof by showing that $\mathfrak{A} / \mathfrak{A}_{0}$ contains no proper ideal of $C\left(S_{0}\right)$. If the contrary is true, then $\mathfrak{A} / \mathfrak{A}_{0}$ contains all continuous functions on $S_{0}$ van- 
ishing on a certain proper subset $S_{1}$ of $S_{0}$. Now an arbitrary extension to $S$ of a function on $S_{0}$ belonging to $\mathfrak{X} / \mathfrak{A}_{0}$ is in $\mathfrak{A}$, because $\mathfrak{A}_{0}$ contains all continuous functions vanishing on $S_{0}$; hence $\mathfrak{A}$ itself contains all functions vanishing on $S_{1}$. For each such function $\phi$ it is obvious that $\phi \cdot \mathfrak{S} \subset \mathfrak{A}$, and so $\phi \in \mathfrak{A}_{0}$. Thus $\phi$ vanishes on $S_{0}$ instead of merely on the smaller set $S_{1}$. This does not hold for all $\phi$ if $S_{1}$ is properly contained in $S_{0}$, and the contradiction proves that $\mathfrak{A} / \mathfrak{A}_{0}$ contains no proper ideal of $C\left(S_{0}\right)$.

We have shown that if $\mathfrak{A}$ is a maximal subalgebra of $C(S)$, containing scalars and separating points, then $\mathfrak{A}$ consists precisely of the extensions to all of $S$ of some maximal subalgebra $\mathfrak{B}$ of $C\left(S_{0}\right)$, where $S_{0}$ is a closed subset of $S ; S_{0}$ can be so chosen that $\mathfrak{B}$ is an integral domain. The opposite construction is quite obvious: the set of all extensions to $S$ of the functions in a maximal subalgebra $\mathfrak{B}$ of $C\left(S_{0}\right)$, where $S_{0}$ is a closed subset of $S$, forms a maximal subalgebra of $C(S)$. The nature of such extensions depends on the topology of $S$, and it may not be simple to describe $\mathfrak{A}$ in terms of $\mathfrak{B}$; nevertheless that is a purely topological problem, and we can be content to discuss maximal algebras without divisors of zero.

THEOREM 4. Let $\mathfrak{A}$ be a maximal subalgebra of $C(S)$ without divisors of zero. If an element of $\mathfrak{A}$ vanishes on a nonempty open set, then it vanishes identically.

Suppose $\phi$ in $\mathfrak{A}$ vanishes on a neighborhood; then we can find a continuous function $f$ which is not zero everywhere but such that $\phi \cdot f=0$. Since $\mathfrak{A}$ has no divisors of zero, $f \notin \mathfrak{A}$. It follows that polynomials in $f$ with coefficients in $\mathfrak{A}$ form a dense subset of $\mathfrak{C}$ (as we have observed before); and since $\phi \cdot f$ is zero, the product of such a polynomial by $\phi$ lies in $\mathfrak{A}$. So $\phi \cdot \mathfrak{S} \subset \mathfrak{A}$, and if $\phi \neq 0$, then $\mathfrak{A}$ contains a nontrivial ideal of $\mathfrak{E}$. That is, $\mathfrak{A}$ contains all functions vanishing on a certain proper closed subset of $S$, and so $\mathfrak{A}$ has divisors of zero. This contradiction shows that $\phi$ must have been the zero function.

THEOREM 5. If $\phi$ is in $\mathfrak{A}$ and is real, then $\phi$ is constant.

For any integer $n, e^{i n \phi}$ can be written as a uniformly convergent power series in $\phi$; hence the function $e^{i n \phi}$ belongs to $\mathfrak{A}$. It follows that $\sum_{n=-\infty}^{\infty} a_{n} e^{i n \phi}$ belongs to $\mathfrak{A}$ for any sequence of coefficients satisfying $\sum_{-\infty}^{\infty}\left|a_{n}\right|<\infty$. If $\phi$ is not constant, find a continuous periodic function $f$ with absolutely convergent Fourier series which vanishes on an interval in which $\phi$ takes values, but which is not zero for all values assumed by $\phi$. Then $f(\phi)$ is a function of $\mathfrak{A}$ and vanishes on an open 
set but not identically. This is impossible, so that $\phi$ is constant as asserted.

To these results we add one more which seems suggestive, but which we have not been able to apply.

TheOREM 6. Let $\mathfrak{A}$ be a maximal subalgebra of $C(S)$ without divisors of zero. Then $\mathfrak{A}$ is integrally closed in the following sense: if $\phi$ and $\psi$ are functions of $\mathfrak{A}$ which do not vanish identically and such that $\phi=f \cdot \psi$ for some CONTINUOUS function $f$, and if $f$ satisfies an equation

$$
f^{n}+\alpha_{1} f^{n-1}+\alpha_{2} f^{n-2}+\cdots+\alpha_{n}=0 \quad\left(\alpha_{j} \in \mathfrak{A}\right),
$$

then $f$ belongs to $\mathfrak{A}$.

If $f$ does not belong to $\mathfrak{A}$, the set of all polynomials in $f$ with coefficients from $\mathfrak{A}$ is dense in $\mathfrak{E}$. By virtue of the relation satisfied by $f$, every polynomial in $f$ is equal to a polynomial of degree at most $n-1$. Clearly for such a polynomial $P$ we have $\psi^{n-1} \cdot P \in \mathfrak{A}$. It follows, because $\mathfrak{A}$ is closed, that $\psi^{n-1} \cdot \mathfrak{C} \subset \mathfrak{A}$, so that $\mathfrak{A}$ contains a nontrivial ideal of $\mathfrak{E}$. But this implies that $\mathfrak{A}$ has divisors of zero, which is not the case, and the truth of the theorem follows.

Further progress depends on finding information about the space of maximal ideals of $\mathfrak{A}$. In the algebras constructed by Wermer, for example, the space $S$ is the boundary of a Riemann surface whose points correspond to the maximal ideals of $\mathfrak{A}$. It seems essential at this point to introduce a restriction on the space $S$; but even in the simplest cases further progress is difficult. ${ }^{2}$

\section{REFERENCES}

1. J. Wermer, On algebras of continuous functions, Proc. Amer. Math. Soc. vol. 4 (1953) pp. 866-869.

2. - Algebras with two generators, Amer. J. Math. vol. 76 (1954) pp. 853-859.

3. - Subalgebras of the algebra of all complex-valued continuous functions on the circle, Amer. J. Math. vol. 78 (1956) pp. 225-242.

YALE UNIVERSITY

2 We have assumed that $\mathfrak{A}$ and $\mathfrak{S}$ are Banach algebras of functions with the topology of uniform convergence. Our results hold for a more general class of algebras, and it is not difficult to trace the properties of $C(S)$ which are essential in the proofs. 